\title{
STATISTICAL PROPERTIES OF DENSE MOLECULAR CLOUDS IN THE GALACTIC CENTER REGION
}

\author{
ATSUSHI MIYAZAKI AND MASATO TSUBOI \\ Institute of Astrophysics and Planetary Sciences, \\ Ibaraki University, Mito, Ibaraki, 310, Japan
}

\section{Data and Analysis}

Data : We used the Galactic center region survey data in CS $J=1-0$ with the Nobeyama 45-m telescope (Tsuboi, Handa, and Ukita 1997).

Cloud Definition : We identified molecular clumps in the positive longitude region of about $\sim 1 \operatorname{deg}^{2}\left(+0^{\circ} 10^{\prime}<l<+1^{\circ} 42^{\prime}\right.$ and $\left.-25^{\prime}<b<+25^{\prime}\right)$ within all survey region.

We have adopted following criteria : A local peak that was higher than $5 \sigma$ level in each cannel map (The channel width is $5 \mathrm{~km} / \mathrm{s}$.) is a clump candidate. For each candidate, we made four position-velocity maps $\left(\theta=0^{\circ}, 45^{\circ}\right.$, $\left.90^{\circ}, 135^{\circ}\right)$, passing through the peak position. We checked whether a clump candidate is separated from others on each of four P-V map.

The LTE mass was estimated by assuming the local thermal equilibrium (LTE) and that the CS emission is optically thin, that the fractional abundance of CS is $\mathrm{X}(\mathrm{cs})=10^{-8}$, and that the excitation temperature is $T e x=50 \mathrm{~K}$.

\section{Results}

We identified 60 molecular clumps in this region. The identified molecular clumps was with mass range between $10^{3}$ and $10^{5} \mathrm{M}_{\odot}$.

Line width-Size relation

On the relation between the velocity line width and the radius of the clouds, the velocity line widths of the Galactic center clouds are about five times larger than those of the Galactic disk clouds (Figure 1(a)). And on the relation between the velocity line width and the mass of the clouds, the line widths of the Galactic center clouds are significant larger than those of the Galactic disk clouds, too. 

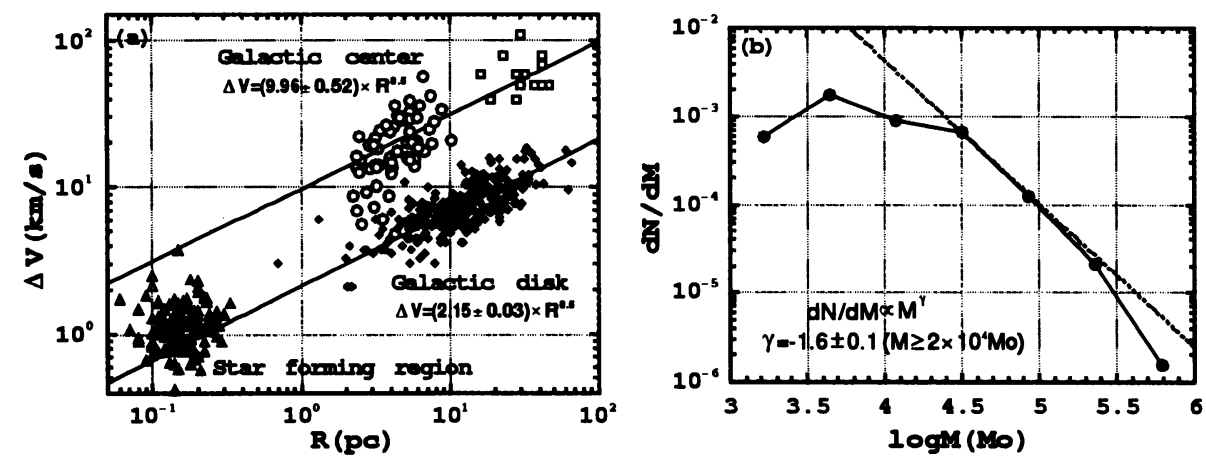

Figure 1. (a) Velocity line width $\Delta \mathrm{V}$ - radius $\mathrm{R}$ relation for the Galactic center clouds (open circles: this work; open squares: Oka et al.1997), the Galactic disk clouds (filled circles: Solomon et al.1987) and the cores in star forming region (open triangle: Orion A GMC,Tatematsu et al.1993). (b) The mass spectrum for Galactic center clumps identified from our data

\section{Virial mass-LTE mass relation}

The virial theorem masses of the Galactic center clouds are about a order of magnitude larger than the LTE masses. These indicate that these molecular clouds is not the virial equilibrium. These are consistent with the results for larger size clouds in the Galactic center from CO $J=2-1$ (Oka et al.1997).

If the molecular clouds in the Galactic center is in equilibrium, mechanisms in order to bound the molecular cloud, e.g. external pressure or magnetic field, are probably needed, except for self-gravity. For example, the expected external pressure in the Galactic center is Pext $/ \mathrm{k}=5.0 \times 10^{7}\left(\mathrm{~cm}^{-3} \mathrm{~K}\right)$ from the virial theorem exerted by a external pressure, and the expected magnetic field is $\mathrm{B}=1.5$ (mGauss) assuming the equipartitions.

\section{Mass Spectrum}

The mass spectrum for Galactic center clump identified from our data is following, (Figure 1(b))

$$
\frac{d N}{d M} \propto M^{-1.6 \pm 0.1}\left(M \geq 2 \times 10^{4} M_{\odot}\right)
$$

This index was similar to one obtained in the other regions.

\section{References}

Tsuboi,M., Handa,T., and Ukita,N. (1997), ApJS, submitted

Oka,T., Hasegawa,T., Hayashi,M., Handa,T., and Sakamoto,S. (1997), ApJ , in press

Solomon,P.M., Sanders,D.B. and Scoville,N.Z. (1987), ApJ, 319, 730

Tatematsu,K. et al. (1993), $A p J, 404,643$ 